\title{
A robot for welding inspection in offshore mooring chains
}

\author{
A. Hernandez, O. Altuzarra, V. Petuya, Ch. Pinto and E. Amezua \\ Department of Mechanical Engineering, University of the Basque Country \\ UPV/EHU,Bilbao,Spain, e-mail: victor.petuya@ehu.es
}

\begin{abstract}
In this paper, the authors present the CIRUS robot. This automatic system uses a combination of pulse-echo and pitch and catch ultrasonic strategies for the inspection of the welded area of mooring chain links in an automatic way. The robot is divided in three parts: global positioning subsystem, local positioning subsystem and inspection subsystem. Additionally, a data acquisition subsystem processes the results of the verification and produces a report for the inspector allowing the traceability of the product for assuring the welding quality.
\end{abstract}

Key words: mooring chain, robotic inspection, ultrasonic strategy.

\section{Introduction}

For the manufacturing of the mooring chains, each link is assembled to the previous one in in the production line. First, the link is heated in a furnace, then bent and finally welded by the flash butt technique. In this kind of chains, the requirements in terms of loads and fatigue are very challenging considering the environment in which they operate. The inspection procedures to be applied to the finished weld can be found in the certification manuals [1], [2]. These inspection procedures cover the usual static tests and also other test mechanical tests. Among them, every link of the chain is subjected to the inspection of the welding plane. Every welding has to be inspected in order to prevent possible fatigue failures caused by defects in the welding plane.

Nowadays, in most companies the Ultrasonic Testing (UT) is done manually by specialized staff. In the case under study, to cover the complete welding plane, it is necessary to perform a scanning in which the transducer has to be moved axially to the link. This motion is repeated around the complete circumference of the link in order to cover all the section diameters. As the process is manual, it not possible to stablish an accurate correspondence between the defect signal and the flaw in the welding. Furthermore, the repeatability of the inspection depend on the experience and ability of the technician. 
In the case under study the method currently implemented is manual and uses the ultrasonic pulse-echo strategy. An ultrasonic transducer is used in accordance with Standard practice ASTM E587 [3]. The procedure uses shear waves with angle $45^{\circ}$ and sampling frequency of $2.25 \mathrm{MHz}$. If a reflection is detected in the transducer, the distance from the defect to the surface is calculated measuring the vertical distance between the transducer and the welding plane. It has to be taken into account that the assembled chain has a connection of the upper and lower links that constrains the accessible surface. Due to the size of the transducer, there is a problem to position it properly in the upper and lowest locations of the inner part of the link. In consequence, it may produce some blacked areas to inspection on the plane of welding for the smaller chain diameters.

This paper presents a novel robotic system specifically focused for the inspection of the weldings of links for mooring chains. The concept has been optimized in terms of simplicity and minimum number of actuators. No current nondestructive testing (NDT) performs the required synchronized inspection motions. Thus, the proposed design is able to guide automatically the transducers combining both inspection motions on the link avoiding any interference with the other chain links [4].

\section{Technical requirements of the robotic system}

The main geometrical-kinematic specifications of the design are:

- Motion geometry of the sensors.

- Ability for inspection in both sides of the welding plane.

- Robustness to manage with diameter irregularities of $10 \%$ in the inspection plane.

- Work ability for different chain diameters.

- Adjustability for different heights of the welding plane.

- Versatility for inspecting two production lines simultaneously.

- Required inspection velocity: 2 min per link.

Also, during conceptual design, it has to be considered the following: ergonomy, safety, calibration process, cost, reliability, low-cost maintenance and robustness.

Considering all these requirements, the traditional inspection strategy of pulseecho using a beam of shear waves at $45^{\circ}$ is modified introducing a new strategy combining pulse-echo and pitch-catch using longitudinal waves at $30^{\circ}$ and sampling frequency of $5 \mathrm{MHz}$. The pulse-echo strategy is more efficient in the external part of the welding plane (sensors at low height with respect to the welding plane). The pitch-catch strategy is more efficient in the central area of the inspection plane. This latter strategy consists on the use of two transducers, located diametrically opposed, in transmission-reception mode. Then, if the beam emitted meets a defect, it reflects in the direction where the receiver captures the wave. 


\section{CIRUS robot}

The concept presented in this paper, is a system that is manually operated by the technician in order to be fixed to the link to be inspected. Once it is fixed and positioned, it manages the inspection in a complete automatic way. Thus, the robot can be divided into three different subsystems,

- The global positioning subsystem that assists the operator in the approach to the link.

- The local positioning subsystem that fixes the system to the link. Thus, the robot is rigidly clamped to the link.

- The inspection subsystem that synchronizes the motion of the transducers for the ultrasonic inspection of the welding plane of the link.

Additionally, there are other systems as the control system and the water and compressed air circuits. These systems will not be presented here.

\subsection{Global positioning subsystem}

It allows the robot to move between two production lines. Also, it absorbs possible abrupt motions in the chain line. For this reason, the robotic device is suspended with a crane from a carrier system. This system is responsible of the translation of the robot in the three spatial directions.

For the vertical motion (along the chain line) in order to face adjusts the robot to the corresponding chain link we propose to use a commercial system (zerogravity device). For the lateral motions, are obtained using a rail fixed to the roof of the manufacturing facilities.

\subsection{Local positioning subsystem}

This subsystem is designed for,

- Adjusting to the diameter of the link.

- Aligning of the robotic system to the plane to be inspected.

- Centring of the transducers with respect to the axis of the link.

- Being the mount of the inspection mechanisms.

The system (Fig. 1) has two pneumatic grippers, adjustable in axial position, so that they can clamp the robot to the chain component. The grippers are provided with specially designed fingers to hold tight the link keeping for inspection its axis coincident with the robot-axis. An adjustable stop ensures the adequate distance from the axis for centring when the robot device is introduced in the link. Before closing the grippers, a laser guide emitted by the robot assists the user to pose the robot vertically with respect to the inspection plane with the aid of a spring on the 
suspension cable. Clamping and centring are simultaneous. For different link diameters, the grippers can be adapted in height and the mechanical stops are set axially.

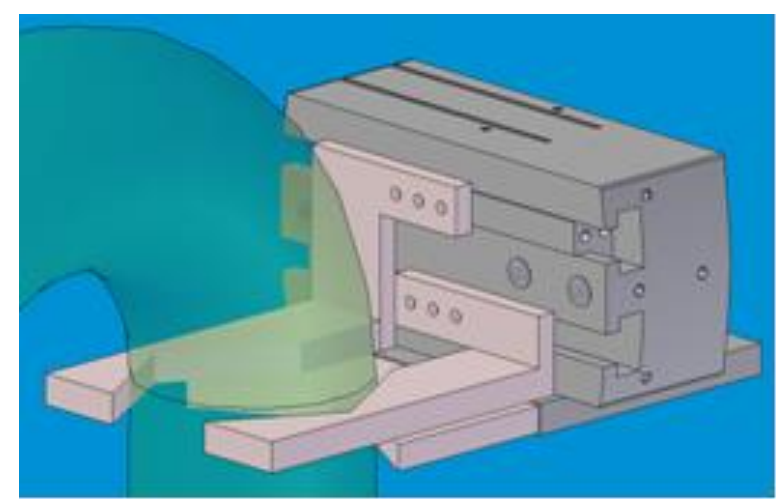

Fig. 1 Local positioning subsystem.

\subsection{Inspection subsystem}

The inspection technique proposed needs a synchronized axial motion of the sensors. The vertical motion required $h$ is 0.577 times the diameter of the link (for a beam emission angle $\alpha$ equal to $30^{\circ}$ ). Additionally, two extra transducers will be mounted symmetrically to the inspection plane in order to ensure redundancy in the inspection process. These extra sensors will move fully synchronized with their pairs. To assure it, each pair of sensors will be mounted in a common splint. The straight vertical motion of the plate is obtained by means of a planar linkage that will be described below.

The vertical motion permits the inspection along a diameter of the section of the plane. To inspect the whole section of the plane, the sensors will sweep a surface corresponding to a circumferential motion of $180^{\circ}$. Each inspection, is stored in a computer database with full information related each of the links of the chain. In this way, the traceability of each of the weldings is assured. The software for the motion control, defect detection and data storage has been developed by INTERLAB Company and is not the purpose of this paper.

A designed frame contains all the inspection subsystem. It also supports the local positioning subsystem. It contains the control handles, and the fixings for the inspection and local positioning subsystems. Below, an actuated pulley is installed to drive a pinion coupled to the ring gear responsible of the circumferential motion of the transducers. The two linkages responsible of the vertical motion of the sensor's splint are mounted in this ring. For the radial approximation of the sensors to the link, a system based on two movable supports is included. The supports travel 
along a linear guide actuated by two pneumatic actuators.

The synthesis of the linkage responsible of the vertical motion of the sensors has been done in two steps,

1. Selecting a linkage with a coupler point able to draw a quasi-straight trajectory.

2. From the selected linkage, generate a new one with a translational coupler link maintaining the quasi-straight trajectory of the joints.

For the first step (type synthesis), some linkage encyclopaedias have been reviewed [5], looking for linkages containing points with this ability of generating a quasi-straight trajectory. From the different possibilities, many of them based on the four-bar linkage, the selected one has been the Hoeckens linkage named "Conchoidal straight-line mechanism".

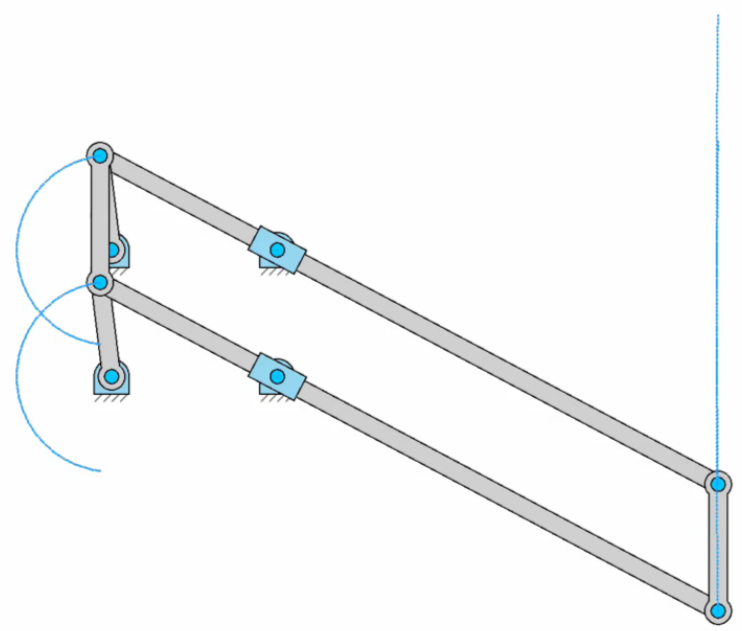

Fig. 2 Mechanism for the vertical motion.

A velocity analysis has been done in order to fulfill the inspection velocity technical requirement. The obtained velocities are low so the inertial effects have not been considered. It is shown how the input angular velocity is quite constant for a constant inspection velocity allowing a simple control of the actuators. This almost constant relationship between the input and output velocities has been one of the main arguments for the selection of the Hoeckens linkage

Finally, the static analysis has been done for a working cycle analysing in successive positions. For this analysis, the resistant loads considered have been the weight of the linkage's elements and mainly the friction force caused by the displacement of the sensors along the travelled irregular surface. It can be observed how the input torque is almost constant along the inspection range, which is other key argument for the selection of the Hoecken mechanism. 
In the second step of the synthesis, a linkage with a coupler link with a straight translational motion has been generated. This coupler link is the splint in which the sensors' mountings are fixed. To obtain the required guiding of the splint, two Hoeckens linkages have been disposed in tandem configuration (Fig. 2). The cranks of the two linkages have been connected by an additional link. To obtain the required input pair, reduction gearboxes have been coupled to each of the motor

The ultrasonic transducers have been mounted in a splint which is the coupler link of the resulting mechanism. A manual adjustment system of the distance between the sensors (to adapt the inspection process to the different link diameters) has been also designed. This system is based on the use of a linear guide for easy adjustment of their relative distance (Fig. 3). Each of the sensors is installed in a mounting allowing two perpendicular rotations. In this way, the sensors can adapt to the roughness of the link's surface. Additionally, this mounting is supported by linear springs in order to assure the continuous contact between sensor and link during the inspection motion.

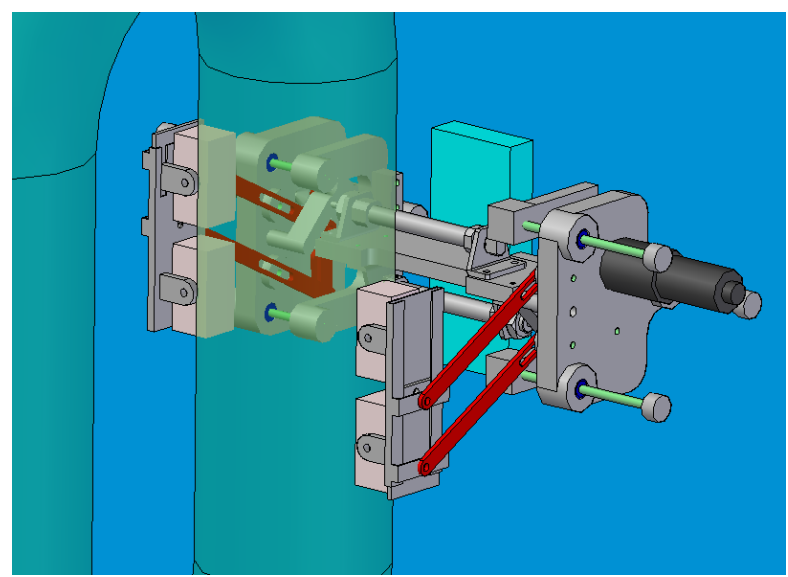

Fig. 3 Inspection subsystem

Finally, in Fig. 4 the CIRUS robot fixed to a link during the ultrasonic testing is shown. 


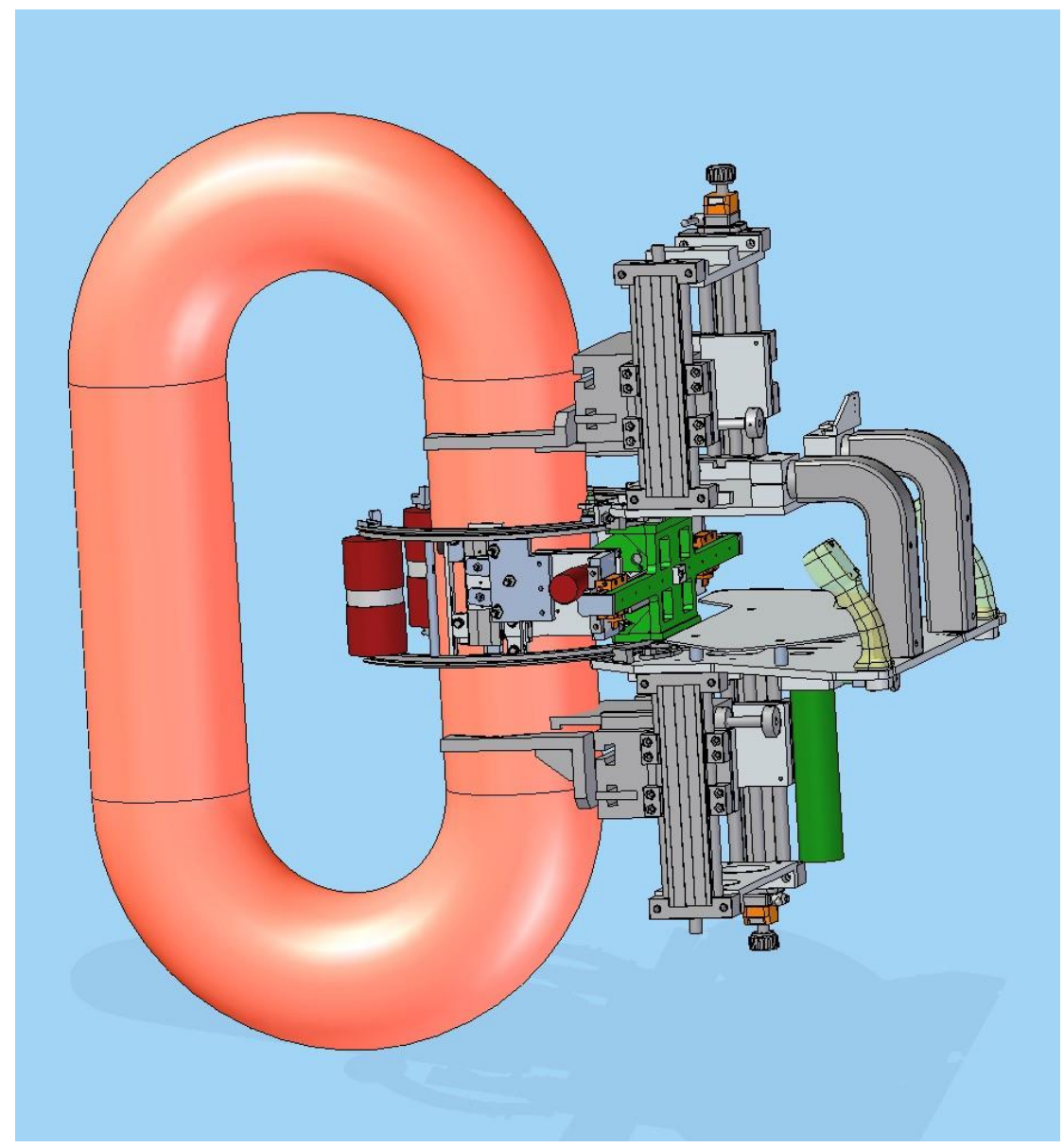

Fig. 4 CIRUS robot.

\section{Conclusions}

A robotic system system for the ultrasonic inspection of the welding plane of links for mooring chains has been presented. In comparison with other commercial NDT equipment, this system has been completely developed focusing on this application. Thus, it can complete the inspection combining the synchronized axial motion of the sensors with a circumferential motion avoiding any interference with the other chain links. In this way, the automatic inspection of the welding plane is done. The traditional inspection strategy of pulse-echo using shear waves is modified introducing a new strategy combining pulse-echo and pitch-catch using longitudinal waves. The project presented has been a great opportunity of 
technological transference between the COMPMECH Research Group of the UPV/EHU and VICINAY MARINE Company. In the web address www.ehu.eus/compmech/contracts-2/robotics-for-ultrasonic-inspection-ofweldings-in-offshore-chains-cirus/ can be found a video of the CIRUS system during the inspection of a chain link.

Acknowledgments The authors wish to acknowledge financial support from VICINAY MARINE S.L, Ministerio de Economía y Competitividad (Project DPI2015-67626-P, MINECO/FEDER, UE), University of the Basque Country (UPV/EHU) (Program UFI 11/29), and Departamento de Educación, Política Lingüística y Cultura of the Regional Government of the Basque Country (Project IT949-16).

\section{References}

1. American Bureau of Shipping. Guide for Certification of Offshore Mooring Chain. Guide (1999).

2. Det Norske Veritas. Certification of Offshore Mooring Chain. Certification notes (1995).

3. Standard practice for ultrasonic angle-beam examination by the contact method. ASTM E587, pp 242-249 (1994).

4. Altuzarra O, Hernandez A, Pinto Ch, Petuya J, Navas J and Lertxundi I. Device for automatic inspection of parts. Patent WO 2010/136614 A1, Madrid (2009).

5. Artobolevski, I. I. Mecanismos en la Técnica Moderna (in Spanish), 6 Vols. MIR, Moscú (1977). 